\title{
Infecção por Leishmania (Leishmania) chagasi em crianças de uma área endêmica de leishmaniose visceral americana na Ilha de São Luis-MA, Brasil
}

\author{
Leishmania (Leishmania) chagasi infection on childhood from endemic \\ area of visceral leishmaniasis in the São Luís-MA, island
}

\begin{abstract}
Arlene J.M. Caldas', Denise R.C. Silva ${ }^{1}$, Célia C.R. Pereira ${ }^{1}$, Paulo Márcio S. Nunes ${ }^{2}$, Benedito P. Silva ${ }^{2}$, Antonio Augusto M. Silva ${ }^{3}$, Aldina Barral ${ }^{4}$ e Jackson M.L. Costa ${ }^{22}$
\end{abstract}

\begin{abstract}
Resumo Realizou-se estudo prospectivo com 648 crianças de zero a cinco anos no município da Raposa-MA, de julho/97 a junho/98, com o objetivo de avaliar as características da infecção por L.(L.)chagasi e verificar se existe associação entre desnutrição e infecção assintomática. Utilizou-se questionário com dados socioeconômicos, ambientais e hábitos de vida; realizou-se Intradermorreação de Montenegro(IDRM) com antígeno de L. amazonensis e Enzyme Linked Immunosorbant Assay(ELISA) para detectar infecção, e exame antropométrico. A prevalência inicial, final e incidência da infeccão foram 18,6\%, 20,6\% e 10,8\% pelo IDRM, e $13,5 \%, 34,4 \%$ e $28 \%$ pelo ELISA, respectivamente. A prevalência da desnutrição crônica (altura/idade) foi 26\%. Não houve associação estatisticamente significante entre desnutrição e infecção assintomática por L. (L.) chagasi. A forma assintomática da doença está presente nas áreas estudadas, necessitando de medidas de controle mais efetivas.
\end{abstract}

Palavras-chaves: Infecção. L. (L.) chagasi. Prevalência. Incidência. Crianças.

Abstract A prospective study was undertaken in 648 children with less than 6 years of age in the municipality of Raposa, Maranhão, Brazil, from June 1997 to June 1998, to evaluate the characteristics of the infection by $\mathrm{L}$.(L.) chagasi and verify if there is an association between malnutrition and asymptomatic infection. A standardized questionnaire was used containing socioeconomic, environmental and behavioral data. Montenegro skin reaction (IDRM) with L. amazonensis and Enzyme Linked Immunosorbent Assay (ELISA) test to detect infection, and anthropometric examination were performed. Initial and final prevalence and incidence of infection were $18.6 \%$, $20.6 \%$ and $10.8 \%$ as measured by IDRM and $13.5 \%, 34.4 \%$ and $28 \%$ according to ELISA. The prevalence of chronic malnutrition was $26 \%$. No association was detected between malnutrition and asymptomatic infection by $\mathrm{L}$. (L.) chagasi. More effective control measures are needed in these areas since asymptomatic infection seems to be on the increase.

Key-words: Infection L.(L.)chagasi. Prevalence. Incidence. Children.

Entre as formas clínicas das leishmanioses, a leishmaniose visceral (LV) ou calazar constitui-se na mais grave, pois, quando não tratada adequadamente, determina elevados índices de letalidade. Encontrase amplamente distribuída no mundo, principalmente em regiões tropicais e subtropicais da Ásia, Oriente Médio, África, e Américas ${ }^{11} 151825$.

No continente americano é conhecida como leishmaniose visceral americana (LVA), tendo maior prevalência em crianças na faixa etária de zero a nove anos, correspondendo a $80 \%$ dos casos detectados, ocorrendo com freqüência em regiões onde a pobreza e desnutrição são comuns 456710111825 .

Embora seja uma doença predominante na área rural $^{9}$, recentemente tem-se estabelecido em áreas urbanas ou periurbanas, onde o vetor encontra condições ambientais propícias para a manutenção do seu ciclo de vida. Os estudos dos casos humanos têm revelado a ocorrência de um processo de urbanização da LVA no Brasil, principalmente em cidades de médio e grande porte do Nordeste, Sudeste e Centro-Oeste, tais como: São Luís-MA, Teresina-PI,

1. Departamento de Enfermagem da Universidade Federal do Maranhão, 2. Núcleo de Patologia Tropical e Medicina Social da Universidade Federal do Maranhão 3. Departamento de Saúde Pública da Universidade Federal do Maranhão, São Luís MA, 4. Departamento de Imunologia da Uiversidade Federal da Bahia Salvador BA.

Endereço para correspondência: Prof ${ }^{a}$ Arlene de Jesus Mendes Caldas. Núcleo de Patologia Tropical e Medicina Social/UFMA. Praça Madre Deus 2, 65025-560 São Luis, MA.

Fax: $5598232-3837 ; 222-5135$

e-mail:ajmc@elo.com.br

Recebido para publicação em 16/7/1999. 
Fortaleza-CE, Natal-RN, Rio de Janeiro-RJ, e Belo Horizonte-MG 6791011181920 .

Sugere-se que tais mudanças sejam atribuídas a fatores inter-relacionados como: transformações ocorridas no ambiente devido ao processo migratório provocados por pressões socioeconômicas; pauperização em função da má distribuição de renda levando ao êxodo rural principalmente nas áreas do Nordeste que sofrem de secas periódicas, agravando a situação nessas áreas, e o aparecimento de novos focos da doença devido ao processo de urbanização crescente nas cidades 610111820 .

Estudos epidemiológicos em áreas endêmicas de LVA têm demonstrado que um percentual de indivíduos apresentam evidências de infecção por Leishmania (Leishmania) chagasi sem história de manifestações clínicas, pois os mesmos revelam altas taxas de indivíduos com sorologia ou IDRM positivos. Cerca de $20 \%$ destes podem apresentar manifestações clínicas, desenvolvendo a chamada forma aguda e os demais podem permanecer assintomáticos ou evoluir para a forma oligossintomática da doença1411 182025 .

No Brasil, de cada seis indivíduos infectados um desenvolve LVA, embora em alguns Estados essa relação seja bem superior, como na Bahia que foi de 18:1 e no Ceará, 11:14510. Nos estudos realizados no Quênia esta relação foi de 5:25.

Diante do exposto, realizou-se o presente estudo com a finalidade de avaliar as características da infecção por $L$. (L.) chagasi em crianças de zero a cinco anos residentes em duas áreas de ocupação recentes no município da Raposa-MA e verificar se existe associação entre desnutrição e infecção assintomática por L. (L). chagasi.

\section{MATERIAL E MÉTODOS}

Realizou-se um estudo epidemiológico prospectivo no período de julho/97 a junho/98, com 648 crianças de zero a cinco anos de idade nas localidades de Vila Nova e Bom Viver no município de Raposa-MA, localizado na llha de São Luís, distando $28 \mathrm{~km}$ da cidade de São Luís, capital do estado do Maranhão. Apresenta área de $63,9 \mathrm{~km}^{2}$ e uma população de 15.075 habitantes distribuídos em 21 localidades, entre elas, Vila Nova com uma população de 2.600 habitantes e Bom Viver com 4.307 habitantes, ambas oriundas de ocupações recentes ${ }^{14}$. A escolha destas duas localidades deveu-se ao elevado número de casos de LVA detectados nos últimos anos pela Fundação Nacional de Saúde(FUNASA), caracterizando-as como áreas endêmicas da doença.

O estudo iniciou-se em julho/97, com a realização de inquérito populacional. Em seguida foi planejado e delineado em duas fases: a primeira, de setembro a outubro/97, com a aplicação de um questionário com perguntas abertas e fechadas sobre os dados demográficos, socioeconômicos, ambientais e hábitos de vida das crianças; realização de exame antropométrico e testes de IDRM e ELISA; e a segunda, de abril a maio/98, com a finalidade de reavaliar o estado imunológico e nutricional das crianças. Durante todo o período de estudo, as crianças com ELISA positivo eram clinicamente avaliadas uma vez por mês. No seguimento do estudo, a vontade e decisão da mãe ou responsável pela criança foram sempre respeitadas. Um termo de consentimento voluntário era assinado.

Realizou-se o teste de IDRM em 639 crianças na primeira, e 572 na segunda fase do estudo, com antígeno preparado no laboratório de Imunologia do Hospital Universitário Prof. Edgar Santos (HUPES),
Universidade Federal da Bahia (UFBA, com formas promastigotas de L. amazonensis (MHOMBr-88-BA125) de acordo com a técnica descrita por Reed e cols ${ }^{22}$. Considerou-se como reação positiva quando um dos diâmetros da enduração era igual ou superior a $5 \mathrm{~mm}$.

A avaliação sorológica foi realizada em 638 crianças na primeira fase, e 572 na segunda fase, pela técnica ELISA ${ }^{22}$, no laboratório de Imunologia do HUPES. A reação foi considerada positiva quando o nível de absorbância era igual ou superior a 0,045 .

O exame antropométrico foi realizado para avaliar a prevalência das diversas formas de desnutrição protéico-calórica e consistiu na medição do peso e altura ou comprimento de cada criança ${ }^{23} 24$.

Analisaram-se os dados no programa EPI-INFO, versão 6.04b, da Organização Mundial da Saúde (OMS). Na análise dos dados referentes à situação nutricional das crianças utilizou-se como referência o padrão do National Center of Health Statistics $(\mathrm{NCHS})^{23} 24$ no programa EPINUT. A comparação do estado nutricional foi realizada somente com as crianças que participaram das duas fases do estudo.

Considerou-se como prevalência inicial as crianças com resultados positivos na primeira fase; prevalência final as crianças com resultados positivos na segunda fase e incidência as crianças com resultados negativos na primeira e positivos na segunda fase. O teste do qui-quadrado foi utilizado para verificar as diferenças entre os eventos do estudo. No caso de freqüências esperadas inferiores a 5 foi utilizado o teste de Fisher. Quando o valor de "p" era menor ou igual a 0,05, considerou-se a diferença estatisticamente significante. 


\section{RESULTADOS}

De acordo com o inquérito populacional, Vila Nova era constituída por 361 famílias e Bom Viver por 574 . Cada família apresentou uma média de 5,2 pessoas e 3,9 filhos. Destes últimos, 1,6 eram crianças na faixa etária de zero a cinco anos de idade.

Das 648 crianças que participaram do estudo, 298 eram de Vila Nova e 350 de Bom Viver. A idade das crianças variou de 11 dias a 71 meses, concentrandose o maior percentual na faixa etária de 0 a 23 meses $(40 \%)$, sendo $48 \%$ do sexo masculino e $52 \%$ do feminino.

No que diz respeito à renda mensal, constatou-se que a maioria das crianças era oriundas de famílias com renda inferior a dois salários mínimos (89\%). A média da renda familiar era de 1,7 salários mínimos por mês. Com referência à escolaridade, $69 \%$ dos pais sabiam ler e escrever.

O tipo de casa predominante era coberta de palha, com paredes de taipa e o piso de chão batido. Segundo os entrevistados não havia coleta pública de lixo, o mesmo era colocado em terreno baldio(29\%), enterrado ou queimado(71\%). O destino final dos dejetos humanos era fossa negra, no quintal ou mato (93\%) (Tabela 1).

No que diz respeito à presença de animais e flebotomíneos no domicílio e vizinhança, mais da

\begin{tabular}{|c|c|c|}
\hline Características & $f$ & $\%$ \\
\hline \multicolumn{3}{|l|}{ Renda familiar mensal } \\
\hline$<1$ a 1,9 salários & 576 & 89,0 \\
\hline$\geq 2$ salários & 72 & 11,0 \\
\hline \multicolumn{3}{|l|}{ Escolaridade dos pais } \\
\hline sabe ler e escrever & 448 & 69,0 \\
\hline não sabe ler e escrever & 200 & 31,0 \\
\hline \multicolumn{3}{|l|}{ Tipo de casa } \\
\hline palha/taipa/chão batido & 581 & 90,0 \\
\hline telha/alvenaria/cimento ou cerâmica & 67 & 10,0 \\
\hline \multicolumn{3}{|l|}{ Lixo } \\
\hline queimado/enterrado & 461 & 71,0 \\
\hline jogado em terreno baldio & 187 & 29,0 \\
\hline \multicolumn{3}{|l|}{ Dejetos } \\
\hline quintal/mato & 602 & 93,0 \\
\hline fossa séptica & 46 & 7,0 \\
\hline \multicolumn{3}{|l|}{ Tipo de animais no Domicílio e vizinhança } \\
\hline nenhum & 239 & 37,0 \\
\hline cão & 108 & 17,0 \\
\hline galinha & 245 & 38,0 \\
\hline galinha/cão & 56 & 8,0 \\
\hline \multicolumn{3}{|l|}{ Presença de flebotomíneos no peri e intradomicílio } \\
\hline $\operatorname{sim}$ & 426 & 66,0 \\
\hline não & 222 & 34,0 \\
\hline \multicolumn{3}{|l|}{ Tipo de doença na família e vizinhança } \\
\hline calazar & 32 & 5,0 \\
\hline outras doenças (tuberculose, gripe, dengue, varicela) & 90 & 14,0 \\
\hline nenhuma doença & 526 & 81,0 \\
\hline \multicolumn{3}{|l|}{ Local das necessidades fisiológicas da criança } \\
\hline dentro de casa & 202 & 31,0 \\
\hline fora de casa & 446 & 69,0 \\
\hline \multicolumn{3}{|l|}{ Local do banho da criança } \\
\hline dentro de casa & 112 & 17,0 \\
\hline fora de casa (quintal) & 536 & 83,0 \\
\hline \multicolumn{3}{|l|}{ Local da criança brincar no final da tarde } \\
\hline ao redor da casa & 526 & 81,0 \\
\hline dentro de casa & 122 & 19,0 \\
\hline Total & 648 & 100,0 \\
\hline
\end{tabular}

$\mathrm{f}=$ freqüência 
metade dos entrevistados afirmaram a existência de um ou mais de um tipo de animal como cão e/ou galinha, e de arrupiado, como é conhecido o flebotómo nas áreas estudadas. A LVA foi citada por $5 \%$ das pessoas entrevistradas como doença predominante entre os familiares e vizinhos no último ano (Tabela 1).

As crianças na sua maioria (69\%) realizavam as necessidades fisiológicas fora da casa (quintal ou mato) e mais de dois terços tomavam banho no quintal. Observou-se, também, que $81 \%$ brincavam ao redor da casa ao entardecer (Tabela 1).Quanto ao estado nutricional, detectou-se uma prevalência de $25,5 \%$ na primeira fase e $26 \%$ na segunda, de desnutrição no indicador altura/idade. No indicador peso/altura a prevalência foi de $1 \%$ nas duas fases e no indicador peso/idade foi de $8 \%$ na primeira fase e $10 \%$ na segunda. Não houve diferença estatisticamente significante nas prevalências de desnutrição entre as duas fases do estudo em nenhum indicador (Tabela 2).

Em relação ao teste de IDRM, detectou-se prevalência inicial e final de infecção por L. (L.) chagasi de $18,6 \%$ e $20,6 \%$, respectivamente, porém a diferença não foi estatisticamente significante entre as duas fases $(P=0,420)$. A incidência foi de $10,8 \%$. Quanto ao ELISA, a prevalência inicial e final foram de $13,5 \%$ e $34,4 \%$, respectivamente, entretanto a diferença apresentou-se estatisticamente significante entre as duas fases $(P=0,001)$. $A$ incidência foi de $28 \%$ (Tabela 3 ).
A prevalência inicial do IDRM foi superior à detectada pelo ELISA, sendo a diferença entre ambas estatisticamente significante $(P=0,004)$. Entretanto, a prevalência final e a incidência detectadas pelo ELISA foram superiores às encontradas pelo IDRM $(P=0,001)$.

Quando se verificou a associação entre estado nutricional e infecção assintomática por $L$. (L.) chagasi segundo os testes de IDRM e ELISA, observou-se que entre as crianças com desnutrição crônica (altura/ idade) $5 \%$ e $3,7 \%$, respectivamente, apresentaram positividade aos testes; $0,2 \%$ das crianças com desnutrição no indicador peso/idade apresentou IDRM positivo; e 1,4\% e 1,2\%, respectivamente, apresentaram positividade nos testes quando 0 indicador era peso/idade. Não houve associação estatisticamente significante entre desnutridos e infecção assintomática por L. (L.) chagasi (Tabela 4).

Das crianças que participaram da primeira fase do estudo, 8 apresentaram história pregressa da doença e 6 desenvolveram LVA durante o estudo. A relação infecção/doença foi de 119:1 segundo IDRM e 28:1 de acordo com ELISA. A positividade da reação de hipersensibilidade retardada foi mais elevada nos pacientes com história pregressa de LVA do que naquelas com doença atual. Entretanto a positividade da resposta humoral não mostrou diferença estatisticamente significante quando se comparou pacientes com história pregressa e atual de $\operatorname{LVA}(\mathrm{P}=0,091)$ (Tabela 5).

Tabela 2 - Prevalência da desnutrição nas crianças de zero a cinco anos de Vila Nova e Bom Viver, Raposa-MA, 1998.

\begin{tabular}{|c|c|c|c|c|c|}
\hline \multirow{3}{*}{ Indicadores nutricionais } & \multicolumn{4}{|c|}{ Fases $(\mathrm{n}=572)$} & \multirow{3}{*}{$\mathrm{P}$} \\
\hline & \multicolumn{2}{|c|}{ primeira fase } & \multicolumn{2}{|c|}{ segunda fase } & \\
\hline & $f$ & $\%$ & $f$ & $\%$ & \\
\hline \multicolumn{6}{|l|}{ Altura/idade } \\
\hline desnutrido & 146 & 25,5 & 149 & 26,0 & 0,892 \\
\hline eutrófico & 426 & 74,5 & 423 & 74,0 & \\
\hline \multicolumn{6}{|l|}{ Peso/altura } \\
\hline desnutrido & 6 & 1,0 & 7 & 1,0 & 0,999 \\
\hline eutrófico & 566 & 99,0 & 565 & 99,0 & \\
\hline \multicolumn{6}{|l|}{ Peso/idade } \\
\hline desnutrido & 44 & 8,0 & 55 & 10,0 & 0,293 \\
\hline eutrófico & 528 & 92,0 & 517 & 90,0 & \\
\hline
\end{tabular}

Tabela 3 - Prevalência inicial, prevalência final e incidência da infecção assintomática por L. (L.) chagasi detectada através de IDRM e ELISA, Raposa-MA, 1998.

\begin{tabular}{|c|c|c|c|c|c|c|c|}
\hline \multirow[t]{2}{*}{ Testes } & \multicolumn{2}{|c|}{$\begin{array}{l}\text { Prevalência } \\
\text { inicial }\end{array}$} & \multicolumn{2}{|c|}{$\begin{array}{l}\text { Prevalência } \\
\text { final }\end{array}$} & \multirow[b]{2}{*}{$\mathrm{P}$} & \multicolumn{2}{|c|}{ Incidência $(n=572)$} \\
\hline & $\mathrm{n}^{\circ}$ & $\% \operatorname{pos}^{\star * *}$ & $\mathrm{n}^{\circ}$ & $\%$ pos. & & $\mathrm{n}^{\circ}$ & $\%$ \\
\hline IDRM $^{*}$ & 639 & 18,6 & 572 & 20,6 & 0,420 & 62 & 10,8 \\
\hline$\underline{\text { ELISA }^{* *}}$ & 638 & 13,5 & 572 & 34,4 & 0,001 & 160 & 28,0 \\
\hline
\end{tabular}


Tabela 4 - Indicadores nutricionais e resultados de IDRM e ELISA. Raposa-MA, 1998.

\begin{tabular}{|c|c|c|c|c|c|c|c|c|c|c|}
\hline \multirow{2}{*}{$\begin{array}{l}\text { Indicadores } \\
\text { nutricionais }\end{array}$} & \multicolumn{5}{|c|}{ IDRM (572) } & \multicolumn{5}{|c|}{ ELISA (572) } \\
\hline & pos & $\%$ & neg & $\%$ & $P$ & pos & $\%$ & neg & $\%$ & $\mathrm{P}$ \\
\hline \multicolumn{11}{|l|}{ Altura/idade } \\
\hline desnutrido & 29 & 5,0 & 117 & 20,0 & 0,581 & 21 & 3,7 & 125 & 22,0 & 0,812 \\
\hline eutrófico & 74 & 13,0 & 352 & 61,5 & & 56 & 9,8 & 370 & 64,7 & \\
\hline \multicolumn{11}{|l|}{ Peso/altura } \\
\hline desnutrido & 1 & 0,2 & 102 & 18,0 & 0,999 & 0 & 0,0 & 6 & 1,0 & 0,999 \\
\hline eutrófico & 5 & 0,9 & 464 & 81,0 & & 77 & 34,5 & 489 & 85,5 & \\
\hline \multicolumn{11}{|l|}{ Peso/idade } \\
\hline desnutrido & 8 & 1,4 & 95 & 16,6 & 0,863 & 7 & 1,2 & 37 & 6,5 & 0,791 \\
\hline eutrófico & 36 & 6,3 & 433 & 75,7 & & 70 & 12,2 & 458 & 80,0 & \\
\hline
\end{tabular}

Tabela 5 - História pregressa e atual de leishmaniose visceral americana (LVA) e resultados de IDRM e ELISA. Raposa-MA, 1998.

\begin{tabular}{|c|c|c|c|c|c|c|c|c|c|c|}
\hline \multirow{2}{*}{$\begin{array}{l}\text { História de } \\
\text { LVA }\end{array}$} & \multicolumn{5}{|c|}{ IDRM } & \multicolumn{5}{|c|}{ ELISA } \\
\hline & pos $^{*}$ & $\%$ & neg $^{\star \star}$ & $\%$ & $\mathrm{P}$ & pos & $\%$ & neg & $\%$ & $\mathrm{P}$ \\
\hline Pregressa & 8 & 100,0 & 0 & 0,0 & 0,003 & 1 & 12,5 & 7 & 87,5 & 0,091 \\
\hline Atual & 1 & 16,7 & 5 & 83,3 & & 4 & 66,7 & 2 & 33,3 & \\
\hline
\end{tabular}

\section{DISCUSSÃO}

Os resultados apresentados no presente estudo sobre as condições socioeconômicas, ambientais e hábitos de vida são relevantes na epidemiologia da LVA. Estas condições podem contribuir para que a LVA seja uma das doenças de maior ocorrência nas zonas rurais e periurbanas de algumas cidades, e também para elevação do índice de infecção por L. (L.) chagasi em populações de regiões endêmicas como Vila Nova e Bom Viver no município da RaposaMA, que possuem características de endemicidade da doença4671011 14181925

As condições de moradia eram precárias nas duas localidades. A Pesquisa Nacional por Amostra de Domicílios (PNAD), realizada em 1991, demonstrou que existem diferenças marcantes entre os estados do Nordeste em relação ao tipo de moradia. No Maranhão, dois terços das casas são construídas de taipa ou adobe, enquanto no Rio Grande do Norte, somente uma casa em cada dez é assim construída ${ }^{121617}$. Estudos realizados no Estado do Pará, demonstraram que o maior número de casos humanos de LVA envolveram crianças que residiam em áreas de ocupações. Isto se deveu ao fluxo de pessoas suscetíveis para área, aumento da densidade populacional de flebotomíneos ou o tipo de moradia, cuja construção pode favorecer a entrada do vetor, principalmente nas casas de construções mais abertas, similares às casas das duas áreas por nós estudadas, predominando a construção de taipa e cobertura de palha ${ }^{1120}$. Também foi observado em nosso estudo um processo migratório intenso chegando a influenciar no quantitativo de crianças na segunda fase.
Analisando-se a prevalência inicial e final detectada pelo IDRM, observou-se que não houve diferença significante nas duas fases e a incidência foi menor que a prevalência, demonstrando portanto que o índice de infecção ficou instável durante o estudo. Entretanto, a prevalência final e incidência da infecção detectadas pelo ELISA foram mais elevadas do que as detectadas pelo IDRM, pode-se explicar devido aos anticorpos serem detectáveis mais precocemente que a resposta da reação de hipersensibilidade retardada ${ }^{8}$. Estes dados confirmam o surto epidêmico vivenciado atualmente na Ilha de São Luis, onde a mesma permanece como a principal área problema de LVA no Estado do Maranhão. Dados confirmados pela FUNASA-MA, que registrou no período de janeiro a dezembro de 1998, 297 (61\%) do total de casos da doença no estado, distribuídos por toda a llha. As localidades estudadas, de agosto de 1997 a agosto de 1998, vêm apresentando em média dois casos de LVA humano por mês e Araújo e cols², estudando a composição dos flebotomíneos em Bom Viver, encontraram como espécie mais representativa, a Lutzomyia longipalpis (97\%).

Quando comparou-se o índice de infecção observado em nosso estudo com outros estudos com crianças menores de 15 anos realizados no Ceará $(4,5 \%)$, na Bahia $(16,1 \%)$ e no Maranhão $(46,7 \%)$, observou-se que o nosso índice foi superior aos encontrados nos estudos do Ceará e da Bahia, porém inferiores aos do Maranhão ${ }^{51021}$. Talvez esta diferença seja em função da faixa etária e metodologia utilizadas nos respectivos estudos. Quanto à relação infecção/ doença, observou-se que houve diferença marcante 
entre os dois testes, a infecção foi detectada na proporção de 119:1 no IDRM e 28:1 no ELISA. portanto, bem superior às proporções encontradas no Quênia, Brasil, Bahia e Ceará45 510112125 .

Em relação ao estado nutricional das crianças estudadas, verificou-se que os déficits moderado e severo no indicador altura/idade que retratam a desnutrição crônica, foram superiores ao observado na Pesquisa Estadual de Saúde e Nutrição (PNSN) realizada em 1991 no Maranhão (24\%), e mais do que o dobro em relação ao Brasil (10,5\%) ${ }^{12} 13161723$. Porém, quando se correlacionou estado nutricional e índice de infecção, notou-se que entre os desnutridos o índice de infecção foi mais acentuado nas crianças com desnutrição crônica. Entretanto, somente 6,6\% (IDRM) e 5\% (ELISA) das crianças infectadas apresentavam algum grau de desnutrição, demonstrando que a infecção por $L$. (L.) chagasi ocorre nos indivíduos de áreas endêmicas independentemente do estado nutricional.

Os resultados sobre a história de LVA demonstraram que todas as crianças com história pregressa da doença apresentaram IDRM positivo. Estes dados demonstram que os indivíduos após certo tempo de cura clínica voltam a apresentar resposta imunocelular específica ausente durante a fase ativa da doença. Entretanto, uma criança com IDRM positivo desenvolveu a doença dois meses após a realização do teste discordando do esperado, pois sabe-se que a imunidade celular desempenha um papel mais relevante que os anticorpos na proteção da infecção mediada por leishmânia ${ }^{1322}$. O que pode ter acontecido é que, como a criança tinha um ano e dois meses de idade, o sistema imunológico nesta faixa etária é vulnerável e as condições socioeconômicas também favoreceram a instalação da doença.

Existem estudos mostrando que títulos significativos de anticorpos anti-leishmânia podem ser detectados por um período de tempo relativamente longo após a cura da doença ${ }^{3}{ }^{4}$ e isto pôde ser constatado em nosso estudo quando uma criança após um ano de cura clinica apresentou positividade ao ELISA. A persistência da positividade ao ELISA após a terapêutica específica para LVA, leva-nos a concluir que o resultado da sorologia isolada não pode ser utilizada como critério de cura da doença ou falha terapêutica sem antes realizar uma avaliação criteriosa.

Por outro lado, observou-se que dois terços das crianças que adoeceram nos sete meses de estudo apresentaram positividade ao ELISA com nível de absorvância limítrofe $(0,045,0,046,0,047,0,050)$, porém as que apresentaram níveis bem elevados como 0,200 não desenvolveram LVA. Este fato vem explicar que o teste sorológico isolado não deve ser utilizado para confirmação diagnóstica, apesar de que os anticorpos específicos para antígeno do parasito têm sido utilizados, com sucesso, no diagnóstico da LVA quando associado com a clínica, epidemiologia e o exame parasitológico ${ }^{1125}$.

O elevado índice de infecção por L.(L.) chagasi observado nas localidades estudadas sugere a presença de formas assintomáticas da doença nas crianças de zero a cinco anos de idade. Medidas de controle devem ser efetivadas nas áreas de estudo e direcionadas prioritariamente a esta faixa etária onde observa-se o maior número de casos registrados da doença.

\section{REFERÊNCIAS BIBLIOGRÁFICAS}

1. Andrade TM, Teixeira R, Andrade JF, Pereira C, Carvalho Filho EM. Estudo da hipersensibilidade do tipo retardado na leishmaniose visceral. Revista do Instituto de Medicina Tropical de São Paulo 24:2498-302, 1982.

2. Araújo ACJ, Rebêlo JMM, Carvalho ML, Barros VLL. Composição dos flebotomíneos(diptera, psychodidae) do município da Raposa-MA, Brasil. Área endêmica de leishmanioses. Entomologia y Vectores 7:33-47, 2000.

3. Badaró R, Carvalho EC, Orge MLGO, Teixeira RS, Rocha H. Imunidade humoral e celular em indivíduos curados de leishmaniose visceral. Revista da Sociedade Brasileira de Medicina Tropical 18:77-83, 1985.

4. Badaró R, Jones TC, Carvalho EM, Pedral-Sampaio D, Reed SG, Barral A, Teixeira R, Johnson Jr WD. New perspectives on a subclinical form of visceral leishmaniasis, Journal of Infectious Diseases 154:1003-1011,1986.

5. Badaró $R$ Progressos nas pesquisas de leishmaniose visceral na área endêmica de Jacobina-Bahia 1934-1989. Revista da Sociedade Brasileira de Medicina Tropical 21:159-164,1988.
6. Costa CHN, Pereira HF, Araújo MV. Epidemia de leishmaniose visceral no estado do Piauí, Brasil. Revista de Saúde Pública 24:361-372, 1990.

7. Costa JML, Viana GMC, Saldanha ACR, Nascimento MDSB, Alvim, AC, Burattini MN, Silva AR Leishmaniose visceral no estado do Maranhão, Brasil: a evolução de uma epidemia. Cadernos de Saúde Pública 11: 321-324, 1995.

8. Davies CR, Mazloumi Gaughi AS. Age, acquired immunity and the risK of visceral leishmaniasis: a prospective study in Iran. Parasitology 119:247-257, 1999.

9. Deane LM. Leishmaniose visceral-estudo sobre reservatórios e transmissores realizados no Estado do Ceará. Tese (Livre Docência), Serviço Nacional de Educação Sanitária, Rio de Janeiro, 1956.

10. Evans TG, Vasconcelos IAB, Lima JW, Teixeira JM, McAullife IT, Lopes UG, Pearson RD, Vasconcelos AW. Epidemiology of visceral leishmaniasis in northeast Brazil. Journal Infectious Diseases 166:1124-1132, 1992. 
11. Fundação Nacional de Saúde. Controle, Diagnóstico e Tratamento de Leishmaniose Visceral (Calazar) - Normas Técnicas. Ministério da Saúde, Brasília, 1996.

12. Fundo das Nações Unidas para a Infância (UNICEF). Crianças e adolescentes no Maranhão: saúde, educação e trabalho. Governo do Estado, São Luís MA, 1992.

13. Fundo das Nações Unidas para a Infância (UNICEF). Saúde e nutrição das crianças nordestinas: pesquisas estaduais 19871992, 1995

14. Gama MEA, Barbosa JS, Pires B, Cunha AKB, Freitas AL, Ribeiro IR, Costa JML. Avaliação do nível de conhecimento que a população residente em área endêmicas periurbana e rural, têm sobre leishmaniose visceral, estado do Maranhão, Brasil. Cadernos de Saúde Pública 14:109-118, 1998.

15. Grimaldi Jr G, Thesh RB, McMahon-Pratt D. A review of the geografic distribution and epidemiology of leishmaniasis in the New World. American Journal of Tropical Medicine and Hygiene 41:687-725, 1989

16. Instituto Brasileiro de Geografia e Estatística (IBGE). Perfil estatístico de crianças e mães do Brasil: aspectos de saúde e nutrição de crianças no Brasil, 1989. UNICEF, Rio de Janeiro, 1992.

17. Instituto de Alimentação e Nutrição. Pesquisa Nacional por Amostra de Domicílios-PND Maranhão. Ministério da Saúde, Rio de Janeiro, 1996.

18. Marzochi MCA, Marzochi KBF. Tegumentary and visceral leishmaniasis in Brazil anthropozoonosis and possibilities for their control. Cadernos de Saúde Pública 10:359-75, 1994.
19. Marzochi MCA, Sabroza PC, Toledo LM, Marzochi KB, Tramontano NC, Rangel-Filho, FB. Leishmaniose visceral na cidade do Rio de Janeiro, Brasil. Cadernos de Saúde Pública 1:5-17, 1985.

20. Monteiro PS, Lacerda MN, Arias RR. Controle da leishmaniose visceral no Brasil. Revista da Sociedade Brasileira de Medicina Tropical 27(sup III):67-72, 1994

21. Nascimento MDSB. Epidemiologia da leishmaniose visceral na Ilha de São Luís, Maranhão- Brasil: análise da dinâmica de transmissão e fatores de riscos relacionados ao desenvolvimento da doença. Tese de Doutorado, Universidade Federal de São Paulo- Escola Paulista de Medicina, São Paulo, 1996.

22. Reed SG, Badaró R, Mansur H, Carvalho EM, Lorenço R, Lisboa A, Teixeira R, Johnson Jr WD, Jones TC. Selection of a skin test antigeni for american visceral leishamniasis. The American Journal of Tropical Medicine and Hygiene 35:79-85, 1986.

23. Tonial S. Políticas públicas de assistência alimentar e avaliação das ações de saúde e nutrição: estudo em crianças menores de 5 anos em São Luís, Maranhão. Dissertação de Mestrado, Universidade Federal do Maranhão, São Luís, MA, 1996

24. Victora CG, Huttly SRA, Lombardi C, Voughan JP. Maternal education in relation to early late child health outcomes: findings from a brazilian cohort study. Society Science Medicine 34 899-905, 1992.

25. World Health Organization. Control of leishmaniasis. Word Health Organization, Geneva, 1990. 